\author{
Kildare Miranda • Roberto Docampo • \\ Orlando Grillo • Anderson Franzen • Márcia Attias • \\ Anibal Vercesi · Helmut Plattner · Joachim Hentschel • \\ Wanderley de Souza
}

\title{
Dynamics of polymorphism of acidocalcisomes in Leishmania parasites
}

\begin{abstract}
Growth of Leishmania mexicana amazonensis promastigotes in different culture media resulted in structurally and chemically different acidocalcisomes. When grown in SDM-79 medium, the promastigotes showed large spherical acidocalcisomes of up to $1.2 \mu \mathrm{m}$ diameter distributed throughout the cell. X-ray microanalysis and elemental mapping of the organelles showed large amounts of oxygen, phosphorus, sodium, potassium, magnesium, calcium, and zinc. Immunofluorescence microscopy using antisera raised against a peptide sequence of the vacuolar-type proton pyrophosphatase of Arabidopsis thaliana that is conserved in the Leishmania enzyme, indicated localization in acidocalcisomes. When cells were transferred to Warren's medium, the acidocalcisomes transformed from spherical into branched tubular organelles. The labeling pattern of the vacuolar proton-pyrophosphatase, considered as a marker for the or-
\end{abstract}

K. Miranda · O. Grillo · A. Franzen · M. Attias · W. de Souza $(\bullet)$ Laboratório de Ultraestrutura Celular Hertha Meyer, Instituto de Biofísica Carlos Chagas Filho,

Universidade Federal do Rio de Janeiro,

Av. Brigadeiro Trompovski, s/n., bloco G,

Cidade Universitaria, CEP 21949-900 Rio de Janeiro, Brazil

e-mail:wsouza@biof.ufrj.br

Tel.: +55-21-22602364

Fax: +55-21-22602364

R. Docampo

Laboratory of Molecular Parasitology

and Center for Zoonoses Research, Department of Pathobiology,

University of Illinois at Urbana,

Champaign, IL, USA

H. Plattner · J. Hentschel

Lehrstuhl f. Zellbiologie/Ultrastrukturforschung,

Fachbereich Biology,

Universität Konstanz,

Konstanz, Germany

A. Vercesi

Laboratório de Bioenergética,

Núcleo de Medicina e Cirugia Experimental,

Universidade Estadual de Campinas,

Campinas-SP, Brazil ganelle, changed accompanying the structural changes of the acidocalcisomes, and the enzyme showed an apparently lower proton-transporting activity when measured in digitonin-permeabilized promastigotes. X-ray microanalysis and elemental mapping of these structures revealed the additional presence of iron. Together, the results reveal that the morphology and composition of acidocalcisomes are greatly influenced by the culture con-ditions.

Keywords Leishmania amazonensis - Acidocalcisomes · Elemental mapping - Vacuolar proton pyrophosphatase . Iron uptake

Electronic Supplementary Material Supplementary material is available in the online version of this article at http://dx.doi.org/10.1007/s00418-004-0646-4

\section{Introduction}

An unusual characteristic of trypanosomatid parasites in comparison with mammalian cells is the presence of acidic calcium-rich organelles that have been termed acidocalcisomes. These were first described in Trypanosoma brucei (Vercesi et al. 1994) and Trypanosoma cruzi (Docampo et al. 1995) and then shown to be present in different members of the Trypanosomatidae family, in apicomplexan parasites (reviewed in Docampo and Moreno 1999, 2001; De Souza et al. 2000), in other unicellular eukaryotes such as the slime mold Dictyostelium discoideum (Marchesini et al. 2002) and the green algae Chlamydomonas rheinardtii (Ruiz et al. 2001a), and more recently in the bacterium Agrobacterium tumefaciens (Seufferheld et al. 2003). Acidocalcisomes of some of these cells are characterized by possessing a vacuolartype $\mathrm{H}^{+}$-ATPase and/or a vacuolar $\mathrm{H}^{+}$-pyrophosphatase for proton uptake, a $\mathrm{Ca}^{2+} / \mathrm{H}^{+}$countertransporting ATPase for $\mathrm{Ca}^{2+}$ uptake, and a $\mathrm{Ca}^{2+} / \mathrm{nH}^{+}$antiporter for $\mathrm{Ca}^{2+}$ release (Vercesi et al. 1994; Docampo et al. 1995; Lu et al. 1997, Scott and Docampo 1998; Scott et al. 1998; Rodrigues et al. $1999 \mathrm{a}, \mathrm{b})$. A Na${ }^{+} / \mathrm{H}^{+}$antiporter that may 
participate in $\mathrm{Ca}^{2+}$ release from these organelles has also been described in T. brucei (Vercesi and Docampo 1996; Vercesi et al. 1997; Rodrigues et al. 1999a) and Leishmania donovani (Rodrigues et al. 1999b; Vercesi et al. 2000). X-ray microanalysis in combination with transmission electron microscopy showed that these organelles contain considerable amounts of sodium, magnesium, phosphorus, potassium, calcium, and zinc (Scott et al. 1997; Rodrigues et al. 1999a, b; Miranda et al. 2000, 2004; LeFurgey et al. 2001). Two exceptions are the bloodstream forms of T. cruzi (Correa et al. 2002) and the culture forms of Phytomonas françai (Miranda et al. 2004) that have been shown to contain iron-rich acidocalcisomes. ${ }^{31} \mathrm{P}$ NMR and biochemical analysis have shown that the phosphorus present in these organelles is primarily in the form of pyrophosphate, and short and long chain polyphosphate (Urbina et al. 1999, Moreno et al. 2000, Ruiz et al. 2001b).

Several functions have been attributed to the acidocalcisomes, such as storage of high energy compounds, calcium and other cations, and regulation of intracellular $\mathrm{pH}$ and cellular osmolarity (reviewed in Docampo and Moreno 1999, 2001; De Souza et al. 2000, LeFurgey et al. 2001). Acidocalcisomes have also been shown to have a dynamic distribution during human foreskin fibroblast invasion by Toxoplasma gondii and adopt a collar-like appearance upon initiation of infection (Drozdowicz et al. 2003). It has been postulated that $\mathrm{Ca}^{2+}$ release from acidocalcisomes could be involved in activating the calmodulin-dependent myosin light chain kinases that regulate the actomyosin motor that governs parasite motility and host cell invasion in T. gondii (Drozdowicz et al. 2003).

In this work, we report that the acidocalcisomes of Leishmania amazonensis promastigotes also undergo dynamic structural and compositional changes when grown in different culture media, strongly suggesting a role for these organelles in the adaptation of the parasites to different environmental conditions.

\section{Materials and methods}

Culture methods

Leishmania mexicana amazonensis promastigotes (ATCC 50131 strain) were grown at $28^{\circ} \mathrm{C}$ in SDM-79 medium (JRH Biosciences) supplemented with $10 \%$ heat-inactivated fetal calf serum or in Warren's medium (Warren 1960) supplemented with $10 \%$ heatinactivated fetal calf serum. Cells were collected at intervals of $6 \mathrm{~h}$, fixed, and growth curves were determined with the use of a Neubauer chamber.

\section{Electron microscopy}

Cells were washed in Dulbecco's phosphate-buffered saline (PBS), fixed in Karnovsky, postfixed in $\mathrm{OsO}_{4}$, and embedded in Polybed 812 epoxide resin. Alternatively, cells were washed in PHEM buffer, $\mathrm{pH}$ 6.8, fixed for 7 days in $2.5 \%$ glutaraldehyde + $1 \%$ tannic acid $+1.8 \%$ sucrose in $0.1 \mathrm{M}$ phosphate buffer, rinsed in distilled water, stained en bloc for $2 \mathrm{~h}$ in $1 \%$ uranyl acetate, and embedded in Polybed 812 epoxide resin. Sections were stained for $30 \mathrm{~min}$ in uranyl acetate, for $5 \mathrm{~min}$ in lead citrate, and observed in a Jeol 1200EX electron microscope operating at $80 \mathrm{kV}$.

Imaging of unfixed whole cells

Cells were washed in PBS, pH 7.2, and suspended in PBS. Droplets were applied to 100-mesh Formvar-coated copper grids, allowed to adhere for $10 \mathrm{~min}$, carefully blotted dry, and observed in an energyfiltering LEO EM 912 electron microscope operating at $80 \mathrm{kV}$. Electron spectroscopic images were recorded at an energy loss of $\sim 60 \mathrm{eV}$ with spectrometer slit width of $20 \mathrm{eV}$. Kinetics of the structural reorganization of acidocalcisomes after transferring cells from SDM-79 to Warren's medium was followed using whole cell preparations collected at intervals of $6 \mathrm{~h}$.

Immunofluorescence microscopy

Cells fixed in freshly prepared $4 \%$ formaldehyde were allowed to adhere in poly(L-lysine)-coated coverslips, permeabilized with $0.3 \%$ Triton X-100 for $3 \mathrm{~min}$, and blocked with $50 \mathrm{mM}$ ammonium chloride and $3 \%$ bovine serum albumin (BSA) in PBS. Immunofluorescence was carried out using a 1:100 dilution of polyclonal antibodies raised against the putative hydrophobic loop III of Araibdopsis thaliana vacuolar-type proton pyrophosphatase $\left(\mathrm{V}-\mathrm{H}^{+}-\right.$ PPase; Sarafian et al. 1992) and a fluorescein isothiocyanatecoupled goat anti-rabbit IgG secondary antibody (1:100). Images were obtained in a confocal laser scanning microscope (Zeiss CLSM 310).

\section{Cryoimmunoelectron microscopy}

Cells fixed in freshly prepared $4 \%$ formaldehyde $+0.1 \%$ glutaraldehyde were centrifuged at $14,000 \mathrm{~g}$, infiltrated with $2.3 \mathrm{M}$ sucrose, transferred to specimen freezing supports, and quick frozen by immersion into liquid nitrogen. Supports were then transferred to a cryoultramicrotome (Reichert) and cryosections obtained at a temperature range of -70 to $-75^{\circ} \mathrm{C}$. Sections were collected in 2.3 M sucrose with a platinum loop, placed on Formvar/carboncoated nickel grids, and transferred to $3 \%$ BSA in PBS. Samples were blocked with $50 \mathrm{mM}$ ammonium chloride and 3\% BSA in PBS. Immunolabeling was carried out using a 1:100 dilution of anti-V- $\mathrm{H}^{+}$-PPase antibody and a 10-nm gold-conjugated goat antirabbit IgG secondary antibody (1:100).

\section{Vacuolar-type proton pyrophosphatase activity}

Acidification of internal compartments was followed by measuring changes in the absorbance of acridine orange at the wavelength pair 493-530 nm in an SLM-Aminco DW2000 dual-wavelength spectrophotometer (Palmgren 1991). Cells were incubated at $28^{\circ} \mathrm{C}$ in $2.5 \mathrm{ml}$ standard reaction medium containing $130 \mathrm{mM} \mathrm{KCl}, 2 \mathrm{mM}$ $\mathrm{MgCl}_{2}, 10 \mathrm{mM}$ HEPES buffer, $\mathrm{pH} 7.2$, with $16 \mu \mathrm{M}$ digitonin before addition of $3 \mu \mathrm{M}$ acridine orange. PPi-driven proton uptake by the acidocalcisomes was measured with the addition of $100 \mu \mathrm{M}$ sodium pyrophosphate to the reaction medium containing digitonin-permeabilized cells. The results shown are representative of at least three experiments.

Morphometric analysis

For determination of the volumetric density of the acidocalcisomes, thin sections of glutaraldehyde tannic acid-fixed promastigotes grown in either Warren's or SDM-79 medium were used. Cells were observed and randomly selected in a LEO EM 912 transmission electron microscope equipped with a SIT 66 camera and a SIS image analysis system. Twenty cell profiles of 
each source were acquired, digitized, and measured. Number and absolute volume of acidocalcisomes were estimated by using whole cells cultivated as described above. Absolute volume of acidocalcisomes in cells grown in SDM-79 medium was determined by measuring the diameters of 50 organelles assuming them as spherical units. Absolute volume of acidocalcisomes in cells grown in Warren's medium was determined by multiplying the area of the organelle by the section thickness in serial sections. Variation in the number of each morphological type of acidocalcisomes seen after transferring the cells from SDM-79 to Warren's medium was also determined by counting whole cell preparations. Statistical significance was determined by Student's $t$-test. $P<0.05$ was taken to be significant.

Electron probe X-ray microanalysis and elemental mapping

Energy dispersive X-ray spectra were recorded from the acidocalcisomes of whole cells dried onto Formvar-coated grids. Control spectra were collected from regions adjacent to the acidocalcisomes and from the Formvar film. Specimens were analyzed in a LEO 912 Omega scanning transmission electron microscope. X-rays were collected for $200 \mathrm{~s}$ using a Li-drifted Si-detector (front area $30 \mathrm{~mm}^{2}$ ) equipped with an ATW atmospheric window. The microscope was operated at $80 \mathrm{kV}$ using a tungsten filament, in the scanning transmission (STEM) imaging mode, spot size was $40 \mathrm{~nm}$, and emission current $\sim 10 \mu \mathrm{A}$. Analyses was performed using a Link multichannel energy analyzer and Link ISIS 3.00 software (Oxford Instruments, Wiesbaden, Germany).

Three-dimensional reconstruction

For serial sectioning, blocks of cells prepared using glutaraldehyde/ tannic acid fixation were trimmed and sectioned as described before (Fahrenbach 1984; Attias et al. 1996). Serial micrographs were printed at a final magnification of 26,000 diameters. The cell membrane, the nucleus, acidocalcisomes, and kinetoplast were outlined on each micrograph with distinct colors, and each plane was separately traced using a digitizing table (Numonics 2205) and a three-dimensional reconstruction and morphometry program for serial sections developed by Young et al. (1987). This program provides the perimeter and area of the digitized profiles, and these data were used for calculation of relative volume of cell structures. The resulting data files consisted of contour outlines representing cross-sections of the objects of interest within the volume. The files were transferred to a Silicon Graphics workstation, and surfaces between planes were generated using the software package SYNU (synthetic universe) (Hessler et al. 1992).

\section{Results}

Leishmania amazonensis cultivated in different media have different growth rates

Leishmania amazonensis has been readily cultured as promastigotes in a variety of media at temperatures below $28^{\circ} \mathrm{C}$. Two frequently used media are the SDM-79 medium originally developed for cultivation of $T$. brucei procyclic forms (Brun and Schönenberg 1979) and the Warren's medium originally developed for cultivation of T. cruzi (Warren 1960). Figure 1 shows that parasites cultivated in Warren's medium have a higher growth rate than parasites grown in SDM-79 medium. Cells grown in SDM-79 medium reached the stationary phase approximately within $96 \mathrm{~h}$ whereas in this period cells grown in Warren's medium were still in the mid-log phase. Cells

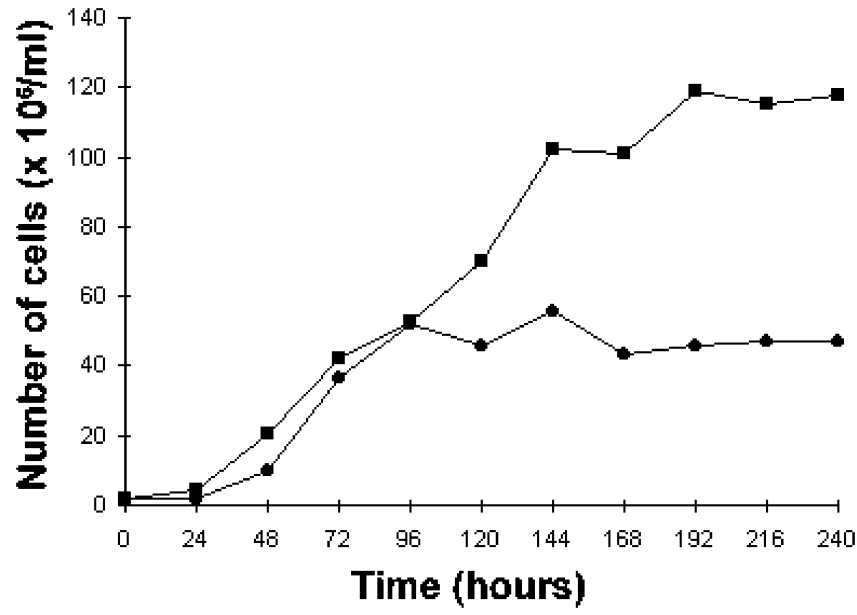

Fig. 1 Cells grown in Warren's medium have a higher growth rate than cells grown in SDM-79 medium. Growth curves of Leishmania amazonensis in SDM-79 medium (circles) and Warren's medium (squares). Curves show that cells grown in SDM-79 medium reach the stationary phase after approximately $96 \mathrm{~h}$ whereas in this period cells grown in Warren's medium are still in the mid-log phase. Cells grown in Warren's medium reach the stationary phase after approximately $192 \mathrm{~h}$

grown in Warren's medium reached the stationary phase after approximately 6 days. Cells grown under both conditions displayed the same shape and motility.

\section{Cells grown in Warren's medium}

show acidocalcisomes of different shapes

Thin sections of promastigotes grown in SDM-79 medium showed large round acidocalcisomes $(\sim 600 \mathrm{~nm}$ in diameter) (Fig. 2A) whereas in cells grown in Warren's medium acidocalcisomes appeared smaller and of irregular shape (Fig. 2B). As seen in Fig. 2A, B, the acidocalcisomes appeared as empty vacuoles as a result of the routine procedure applied for transmission electron microscopy that depletes them of their content (Docampo and Moreno 1999, 2001; de Souza 2000). As it has been described previously (Miranda et al. 2000), a useful method to visualize the acidocalcisomes in their "native" state is the preparation of whole mounts of unfixed cells in electron microscope grids and their subsequent observation with an energy-filtered transmission electron microscope. Under these conditions, acidocalcisomes appear as electron-dense spots. Figure 3 shows that cells grown in SDM-79 medium contained only round acidocalcisomes distributed throughout the cell body and, in some cases, aggregated in the median-posterior region of the cell (Fig. 3A). A large number ( 21/cell, $n=30)$ of very large acidocalcisomes (up to $1.2 \mu \mathrm{m}$ in diameter) could be detected in these cells. In contrast, promastigotes grown in Warren's medium showed a lower number ( 11/ cell, $n=30$ ) of acidocalcisomes which displayed different shapes: some were round, some were slightly elongated, and some were heavily branched (Fig. 3B). Morphometric 


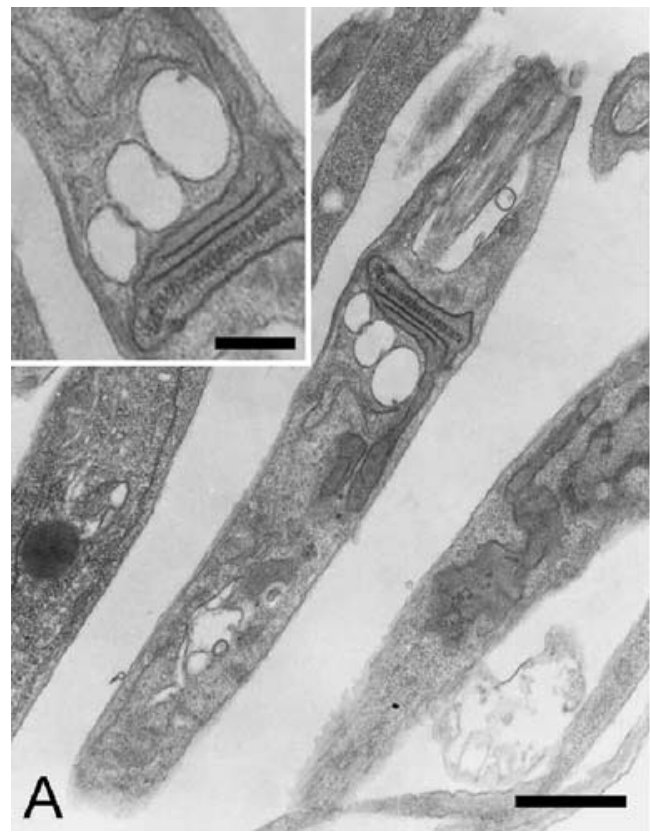

Fig. 2A, B Cells obtained from different sources display acidocalcisomes with distinct ultrastructural characteristics. Thin sections of Leishmania amazonensis cultivated respectively in SDM79 (A) and Warren's medium (B) submitted to conventional glutaraldehyde-osmium fixation. A Cells grown in SDM-79 medium

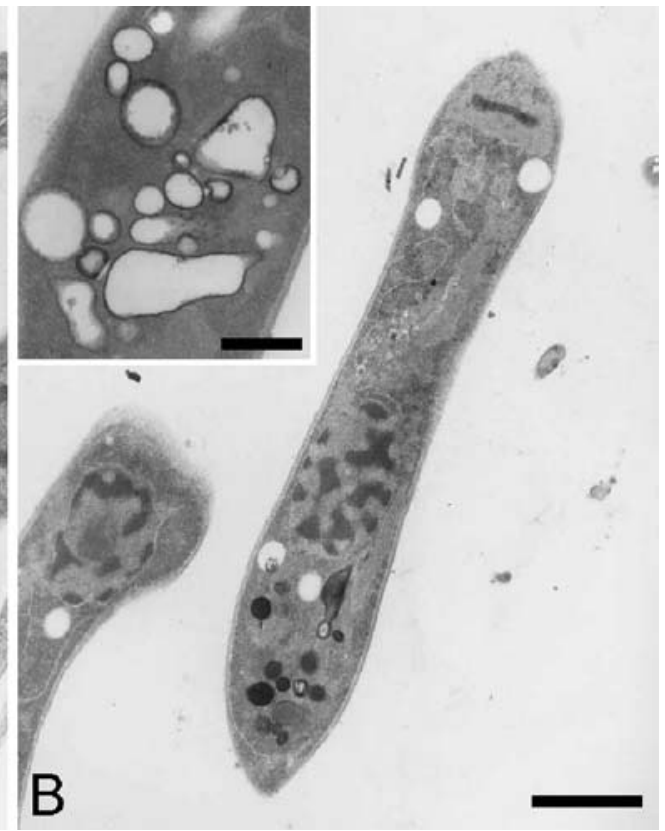

display empty circular vacuoles that correspond to the acidocalcisomes (inset). B Cells grown in Warren's medium display polymorphic acidocalcisomes with an electron-dense material associated to the inner face of their membranes (inset). Scale bars A $1.5 \mu \mathrm{m}$, inset $1 \mu \mathrm{m} ; \mathbf{B} 1.5 \mu \mathrm{m}$, inset $500 \mathrm{~nm}$

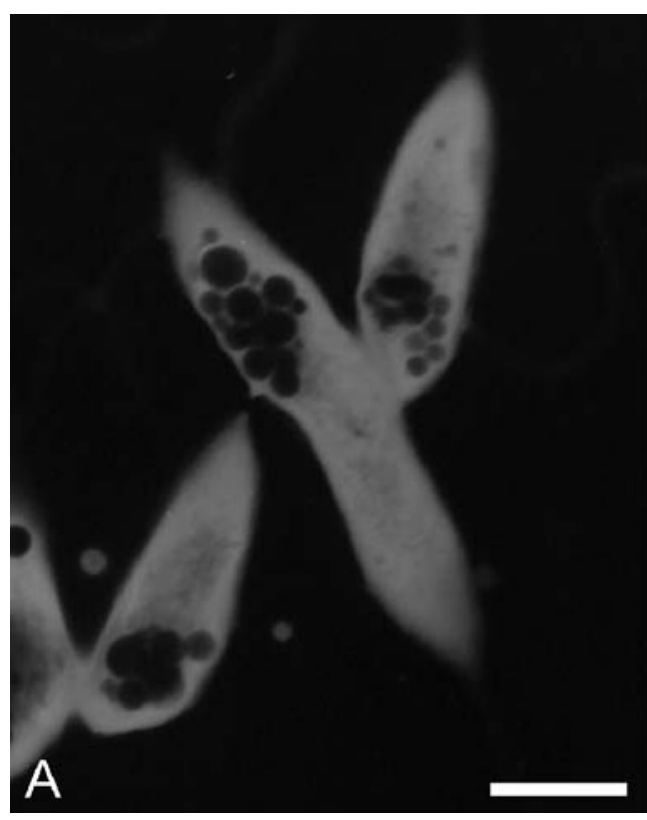

Fig. 3A, B Whole cells show spherical acidocalcisomes in cells grown in SDM-79 and branched acidocalcisomes in cells grown in Warren's medium. Electron spectroscopic images of whole intact cells adhered to Formvar/carbon-coated grids ( $\Delta \mathrm{E}$ between 60 and $80 \mathrm{eV}$ ). A Cells grown in SDM-79 medium. The presence of sev-

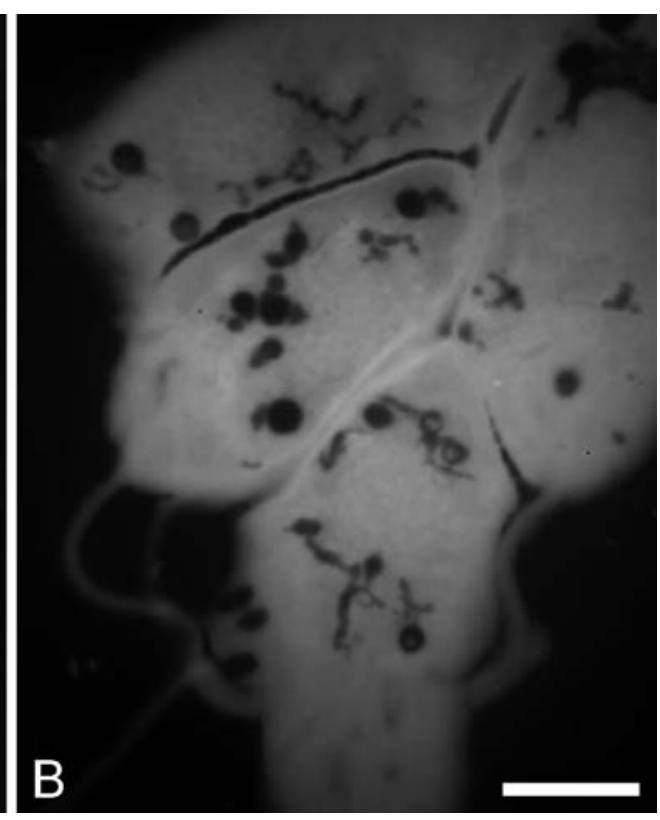

eral spherical acidocalcisomes differing in size and located in different portions of the cell body is easily recognized. Note the presence of large acidocalcisomes. B Cells grown in Warren's medium show polymorphic acidocalcisomes. Scale bars A $4 \mu \mathrm{m}$; B $3 \mu \mathrm{m}$ 
analysis showed that in promastigotes grown in SDM-79 medium the acidocalcisomes occupied $\sim 5.8 \%$ of the cell volume whereas in cells grown in Warren's medium these organelles occupied $\sim 4.9 \%$ of the cell volume.

Acidocalcisomes can change their morphology and fuse to form branched organelles as a result of changes in the culture conditions

We transferred cells grown in SDM-79 medium to Warren's medium and collected them at intervals of $6 \mathrm{~h}$ to follow the kinetics of the transformation process of their acidocalcisomes. Figure 4A shows the acidocalcisomes at the start of the experiment immediately after transference from SDM-79 to Warren's medium $(\mathrm{t}=0)$. All acido- calcisomes displayed a spherical shape. After $6 \mathrm{~h}$ (Fig. 4B, C) some slightly elongated acidocalcisomes were observed in the cells. Interestingly, after $12 \mathrm{~h}$ some acidocalcisomes displayed the central portion of their matrix partially empty (Fig. 4D). In some cells, more than $90 \%$ of the acidocalcisomes showed this appearance. After $24 \mathrm{~h}$ (Fig. 4E-J), acidocalcisomes appeared to fuse with each other, and large, elongated (Fig. 4E arrow), or partially empty acidocalcisomes (Fig. 4F, H arrows) predominated in the cells. After $30-36 \mathrm{~h}$ (Fig. 4K-N), elongated, thin, and branched acidocalcisomes were seen in the great majority of the cells. It is important to note that the structural changes did not occur synchronously in all the cells. Some cells displayed polymorphic acidocalcisomes whereas other cells in the same preparation still showed spherical forms. Therefore, the figures shown are representative of the structural changes observed in the acidocalcisomes of the majority of the cells at a given time. Interestingly, when promastigotes cultured in Warren's medium were transferred back to SDM-79 medium, the acidocalcisomes assumed again a spherical form (data not shown).

Iron is detected in the polymorphic acidocalcisomes when cells are transferred to Warren's medium

X-ray microanalysis of L. amazonensis grown in SDM-79 medium showed that acidocalcisomes contained considerable amounts of sodium, magnesium, phosphorus, potassium, calcium, zinc, and oxygen (Fig. 5A, B), an elemental composition similar to that described previously for acidocalcisomes of $L$. donovani (Rodrigues et al. 1999b) and L. major (LeFurgey et al. 2001). Elemental mapping showed that most of these elements were restricted to acidocalcisomes, demonstrating their important role in ion storage in these parasites (Fig. 5C-K). As seen in Fig. 5K, sulfur was not detected within acidocalcisomes but mostly in the cytoplasm, suggesting a low protein content, or at least a low content of proteins containing sulfur within these organelles. No significant iron content was detected after X-ray microanalysis of the samples (Fig. 5B) or upon elemental mapping of acido- calcisomes (Fig. 5I). X-ray microanalysis of the polymorphic acidocalcisomes of promastigotes grown in Warren's medium (Fig. 6), showed a similar abundance of sodium, magnesium, phosphorus, potassium, calcium, and zinc although with some differences in the stoichiometry of the elements (i.e., relatively lower amounts of magnesium and higher amounts of sodium in promastigotes grown in Warren's medium) (Fig. 6D-K). However, in contrast to the acidocalcisomes of cells grown in SDM-79 medium, the polymorphic acidocalcisomes were shown to contain iron, as detected by X-ray microanalysis (Fig. 6B) and elemental mapping (Fig. 6I).

Localization of the $\mathrm{V}-\mathrm{H}^{+}$-PPase in acidocalcisomes and its proton-pumping activity in promastigotes cultured in different media

Immunofluorescence microscopy using polyclonal antibodies raised against the hydrophobic loop III of $A$. thaliana $\mathrm{V}-\mathrm{H}^{+}$-PPase (Sarafian et al. 1992) showed labeling of large spherical vacuoles in promastigotes grown in SDM-79 medium (Fig. 7A, B) with a similar organization and distribution to the acidocalcisomes detected in the whole cell images depicted in Figs. 3, 4, and 5. Twentyfour hours after their transference to Warren's medium, promastigotes showed labeling of spherical (Fig. 7C, D arrow) as well as polymorphic and elongated structures (Fig. 7C, D arrowheads, E, F) similar to those observed in the whole cell images of Figs. 3, 4, and 6. Promastigotes grown in Warren's medium showed labeling of polymorphic and elongated structures. To confirm whether the structures labeled with fluorescence antibodies corresponded to acidocalcisomes, we performed immunoelectron microscopy of cryosections of promastigotes using the same antibodies. Results showed labeling of large empty vacuoles (Fig. 8A-C), of similar appearance and size to those found using conventional transmission electron microscopy (Fig. 2A) or immunofluorescence microscopy (Fig. 7B, D). Labeling occurred predominantly in the membrane of the organelle, despite the presence of gold particles in the matrix of some acidocalcisomes (Fig. 8B), which might be explained by the presence of soluble pyrophosphatases in these organelles, as occurs in T. brucei (Lemercier et al. 2003). Because a polyclonal antibody was used, it is possible that it has recognized specific sites of the soluble pyrophosphatases that are conserved in the vacuolar-type pyrophosphatase (i.e., PPi binding sites).

In order to investigate the role of the $\mathrm{V}-\mathrm{H}^{+}$-PPase in the acidification of acidocalcisomes of promastigotes cultured in different media, we measured pyrophosphatedependent acridine orange uptake by permeabilized cells. Acridine orange is a tertiary amine that becomes concentrated in acidic compartments within cells and changes both its absorbance and fluorescence properties as a consequence of its accumulation and subsequent dimerization (Palmgren 1991). Addition of pyrophosphate to a reaction medium containing oligomycin and permeabi- 


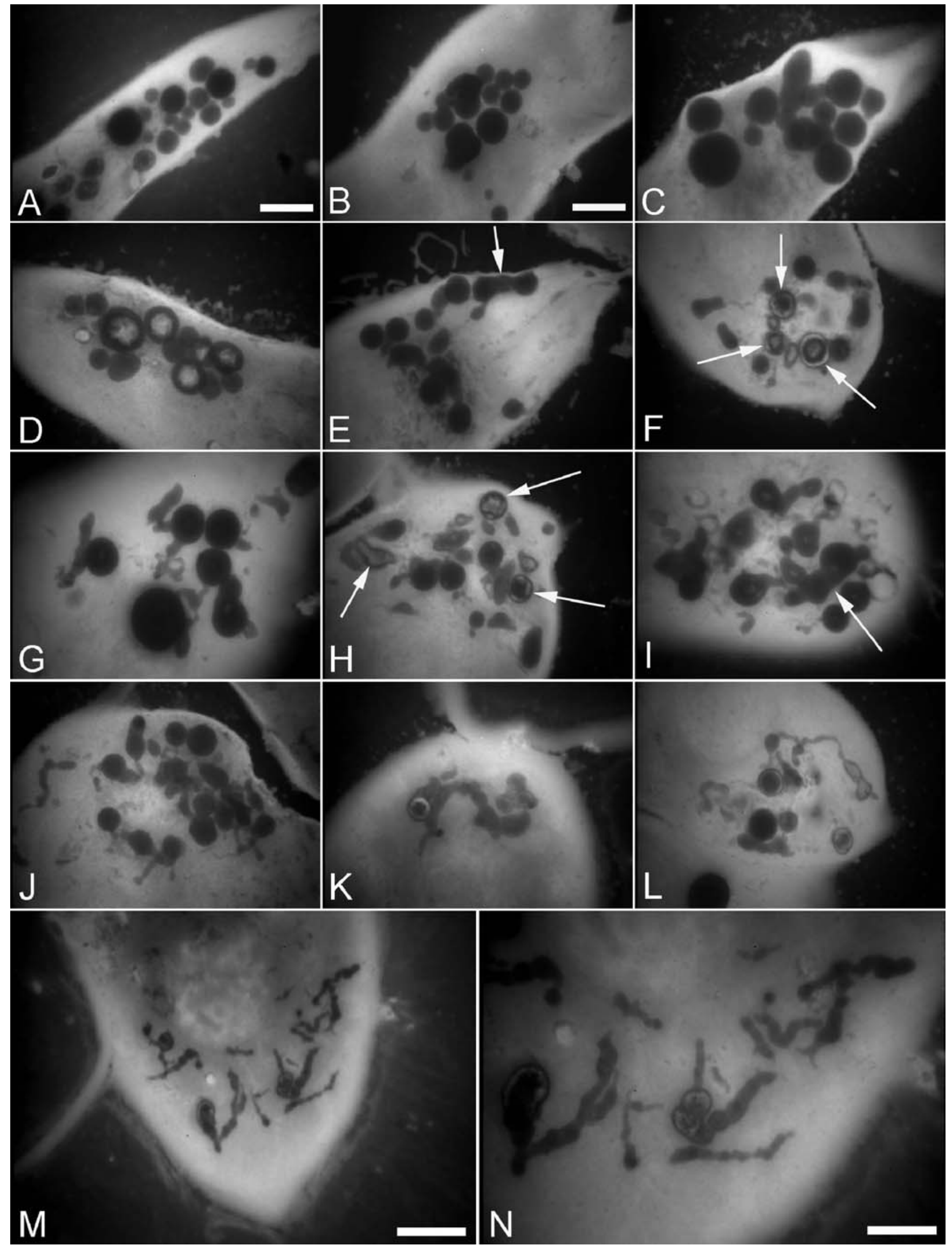


Fig. 5A-K Acidocalcisome of cells grown in SDM-79 medium are the main site for cation storage. A Electron spectroscopic imaging of whole unfixed Leishmania promastigotes $(\Delta \mathrm{E}$ between 60 and $80 \mathrm{eV}) . \mathrm{B}$ Corresponding X-ray spectrum of the acidocalcisome indicated by the arrow in A. Copper peaks in the spectrum came from the grid. Carbon and chlorine signals were similar in the acidocalcisomes and control regions (cytoplasm). C Electron spectroscopic image of a portion of a promastigote $(\Delta \mathrm{E}$ between 60 and $80 \mathrm{eV}$ ). D-K Elemental images of the cell displayed in $\mathbf{C}$ corresponding to: $\mathbf{D}$ phosphorus; E magnesium; $\mathbf{F}$ calcium, $\mathbf{G}$ potassium; $\mathbf{H}$ sodium; I iron; J zinc; and $\mathbf{K}$ sulfur. Note the absence of iron and sulfur in the acidocalcisomes. Scale bars A $2.0 \mu \mathrm{m}$; C $1 \mu \mathrm{m}$; D-K same size as in $\mathbf{C}$
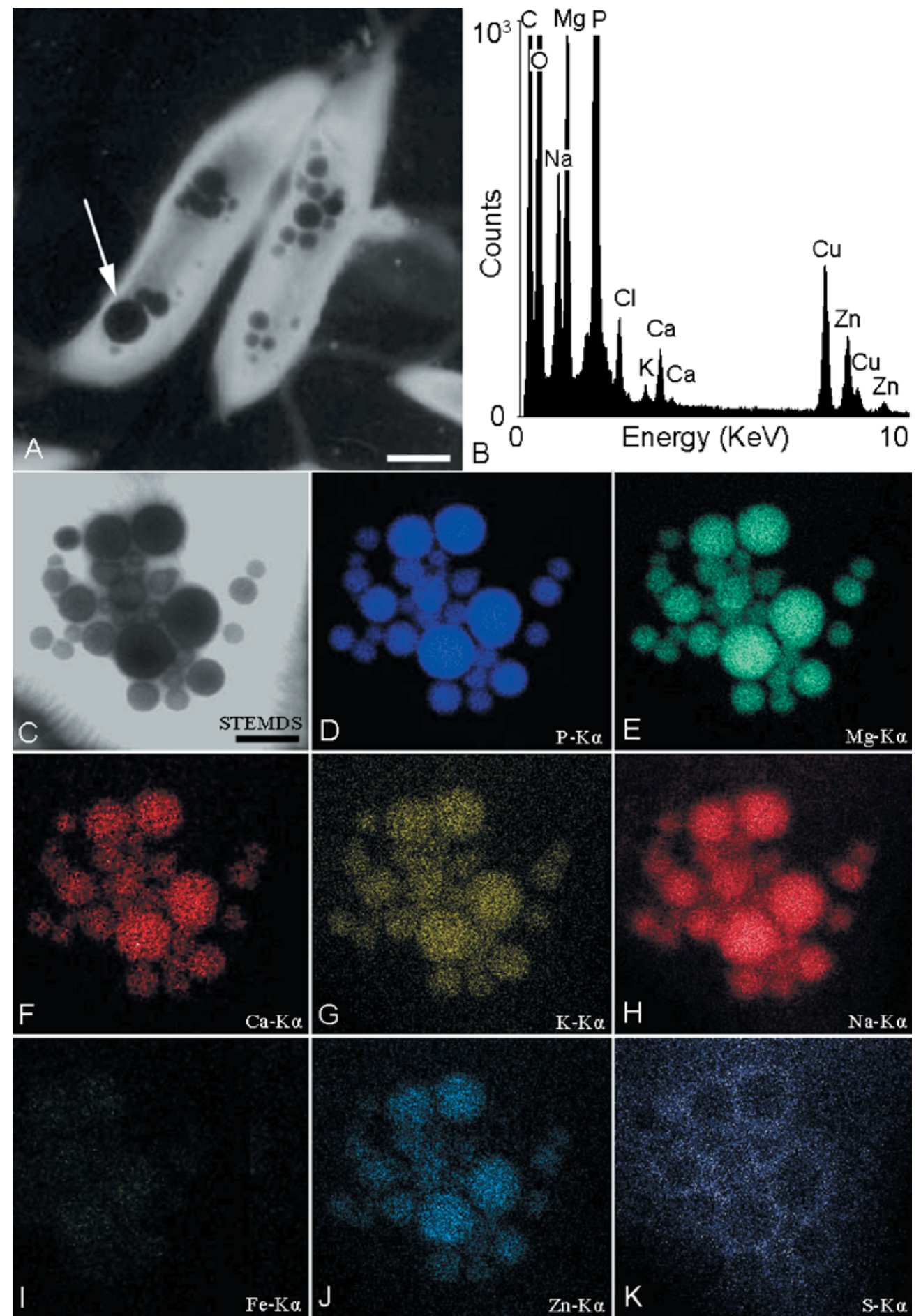

Fig. 4A-N Transference of cells from SDM-79 to Warren's medium triggers polymorphic changes in acidocalcisomes. Spherical (A), large, elongated (E, I, arrows), and partially empty acidocalcisomes $(\mathbf{F}, \mathbf{H}$, arrows) can be seen during the structural reorganization of the organelle. Kinetics of the transformation of spherical acidocalcisomes into polymorphic structures. A $\mathrm{t}=0$ after transferring cells from SDM-79 to Warren's media. Note that all acidocalcisomes are spherical. $\mathbf{B}, \mathbf{C} \mathrm{t}=6 \mathrm{~h} ; \mathbf{D} \mathrm{t}=12 \mathrm{~h} ; \mathbf{E}-\mathbf{J} \mathrm{t}=24 \mathrm{~h} ; \mathbf{K}$ $\mathrm{t}=30 \mathrm{~h} ; \mathbf{L}-\mathbf{N} \mathrm{t}=36 \mathrm{~h}$. Scale bars A $800 \mathrm{~nm} ; \mathbf{B} 500 \mathrm{~nm}$; C-L same size as in $\mathbf{B} ; \mathbf{M} 400 \mathrm{~nm} ; \mathbf{N} 200 \mathrm{~nm}$ lized promastigotes grown in SDM-79 medium caused a significant time-dependent decrease in the absorbance of acridine orange (Fig. 8D trace $a$ ). The addition of nigericin caused a reversal of the absorbance decrease. Similar experiments using the same amount of cells grown in Warren's medium resulted in a lower decrease in acridine orange absorbance (Fig. 8D trace $b$ ), suggesting a lower proton pyrophosphatase activity. 
Fig. 6A-K Presence of iron in acidocalcisomes of promastigotes grown in Warren's medium. A Electron spectroscopic imaging of whole unfixed Leishmania promastigotes $(\Delta \mathrm{E}$ between 60 and $80 \mathrm{eV})$. B Corresponding X-ray spectrum of the acidocalcisomes (A). Note the presence of an iron peak (arrow). Copper peaks in the spectrum came from the grid. Carbon and chlorine signals were similar in the acidocalcisomes and control regions (cytoplasm). C Electron spectroscopic image of a portion of a whole promastigote ( $\triangle \mathrm{E}$ between 60 and $80 \mathrm{eV}$ ). D-K Elemental images of the cell displayed in $\mathbf{C}$ corresponding to: $\mathbf{D}$ phosphorus; E magnesium; $\mathbf{F}$ calcium; G potassium; H sodium; I iron; J zinc; and $\mathbf{K}$ sulfur. Note the presence of iron in the acidocalcisomes (I). Scale bars A $1.0 \mu \mathrm{m}$; C $300 \mathrm{~nm}$; D-K same size as in $\mathbf{C}$
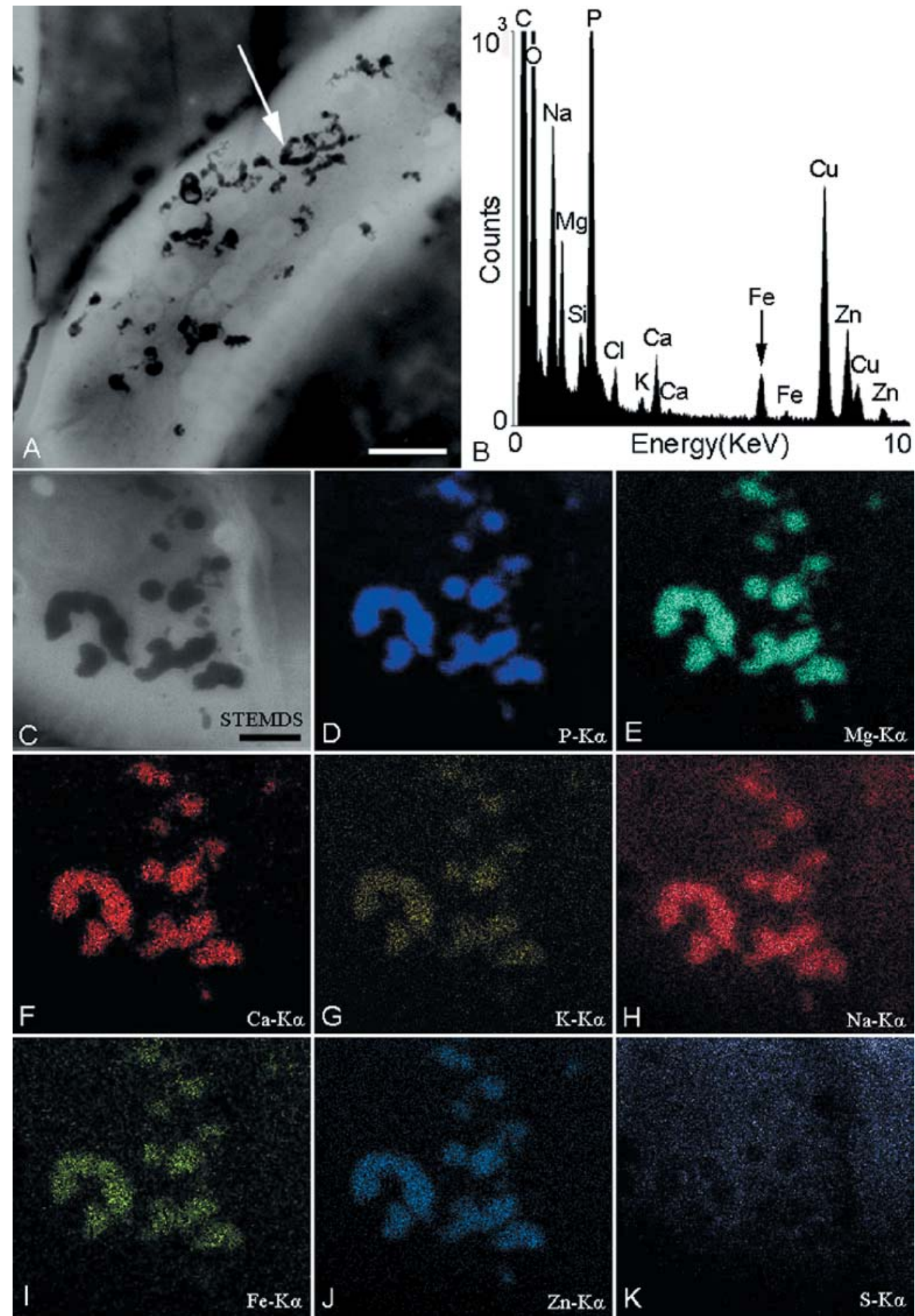

Three-dimensional reconstruction of serial sections show the degree of ramification of acidocalcisomes from promastigotes grown in Warren's medium

The preparation of whole cells dried over Formvar-coated electron microscopy grids is an excellent procedure to preserve acidocalcisomes in their "native" state (Figs. 3, 4, 5, 6). However, when imaging branched forms, the flattening of the sample upon drying could lead to a de- formation of the organelles. This problem is not particularly important to define the morphology of spherical acidocalcisomes, since flattening would still lead to a spherical appearance. Therefore, to overcome this problem, we performed a three-dimensional reconstruction of serial sections of promastigotes grown in different media. The results showed that, as observed in whole cell preparations (Figs. 3, 4, 5), cells grown in SDM-79 medium had large spherical acidocalcisomes, occupying a 
Fig. 7A-F Antibodies raised against a plant vacuolar $\mathrm{H}^{+}$PPase recognize Leishmania structures. Immunofluorescence microscopy using antibodies raised against Arabdopsis thaliana $\mathrm{V}-\mathrm{H}^{+}$-PPase shows labeling of large spherical intracellular structures of Leishmania grown in SDM-79 medium $(\mathbf{A}, \mathbf{B})$, in a disposition similar to the acidocalcisomes observed in whole cells images. $\mathbf{C}, \mathbf{D}$ $\mathrm{V}-\mathrm{H}^{+}$-PPase labeling of cells $24 \mathrm{~h}$ after transferring from SDM-79 to Warren's medium. Note the labeling of both spherical organelles (arrow) and elongated ones (arrowheads). E, F V-H ${ }^{+}$-PPase labeling in cells grown in Warren's medium. Note the labeling in elongated structures. Scale bars A-D $4.0 \mu \mathrm{m} ; \mathbf{E}, \mathbf{F} 3.0 \mu \mathrm{m}$
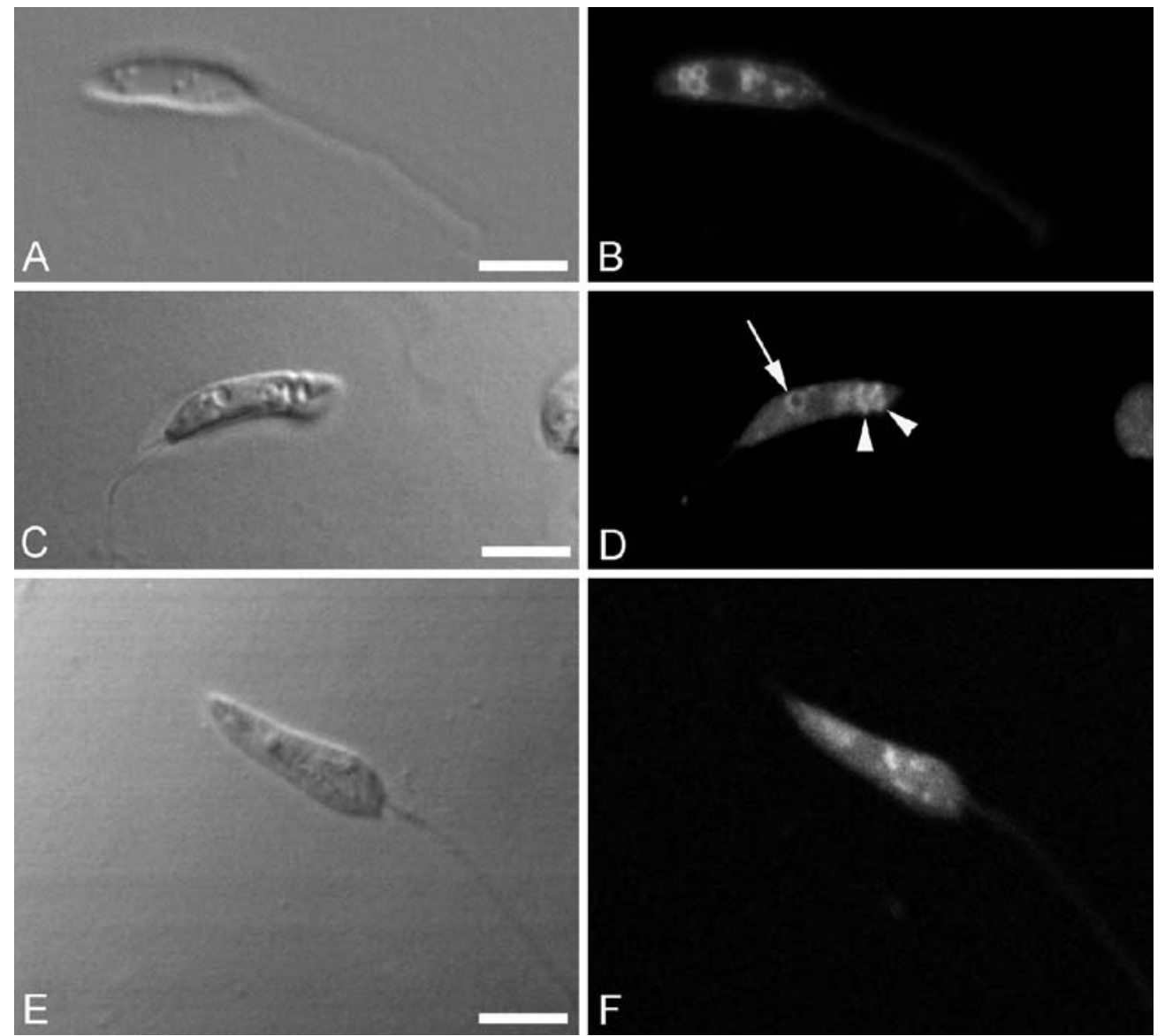

considerable volume of the cell (Fig. 9A). In reconstructed cells grown in Warren's medium it was possible to observe the ramification of the acidocalcisomes (Fig. 9B), especially when observed at different angles (see supplementary material) or through the observation of stereopairs (Fig. 9C, D). In this reconstructed image of promastigotes grown in SDM-79 medium the acidocalcisomes occupied a large volume of the cell, reaching up to $15 \%$ of the total cell volume.

\section{Discussion}

Previous studies have demonstrated functional changes in the acidocalcisomes of $T$. cruzi, such as $\mathrm{Ca}^{2+}$ release and polyphosphate hydrolysis or synthesis, upon changes in ambient $\mathrm{pH}$ and osmolarity (Ruiz et al. 2001b). Structural changes have been detected in the acidocalcisomes of $T$. gondii during invasion of host cells by using immunofluorescence methods (Drozdowicz et al. 2003). In this work, we report that acidocalcisomes of L. amazonensis promastigotes can also undergo structural and compositional changes as detected by immunofluorescence, electron microscopy, X-ray microanalysis, and elemental mapping, when the cells are grown in different culture media. Together these results strongly support a role for these organelles in the adaptation of parasites to different environmental conditions.

Environmental adaptation is an obligate step for the establishment of infection by digenetic organisms. The infection process has important consequences for the cell biology of the host-parasite interaction, involving responses mediated by signaling pathways that coordinate processes involved in cell growth, development, and function (Parsons and Ruben 2000; Lingelbach 2001). In this regard, the adaptation of Leishmania parasites to the different environments encountered in their distinct hosts is a crucial process for the establishment of a successful infection. The ability to adapt to environmental changes is an essential feature of trypanosomatids for the completion of their life cycles. For instance, during the course of infection, the parasites assume different developmental forms in processes that involve several structural changes (reviewed in Handman 2000). Modifications in the shape of the cells, promoted by sliding of the subpelicular microtubules (reviewed in de Souza 2002), changes in the configuration (packing and interaction of maxicirles and minicircles) of the kinetoplast DNA (Guilbride and Englund 1998), shifting of the region where the flagellum emerges from the flagellar pocket, and appearance and disappearance of organelles of the endo-lysosomal system, such as reservosomes in T. cruzi (Porto-Carreiro et al. 2000; Cunha-e-Silva et al. 2002) and megasomes in $L$. 
Fig. 8A-D The V-H ${ }^{+}$-PPase is associated with the Leishmania acidocalcisomes and is more active in cells grown in SDM79 medium. A-C Cryoimmunoelectron microscopy using antibodies against the plant $\mathrm{V}-\mathrm{H}^{+}$-PPase. Note labeling in large empty acidocalcisomes, similar to that observed in thin section preparations of embedded cells. D PPi-driven proton uptake in digitonin-permeabilized cells. Cells $(0.1 \mathrm{mg} / \mathrm{ml})$ were incubated in $130 \mathrm{mM} \mathrm{KCl}$ standard reaction medium containing $1 \mu \mathrm{M}$ oligomycin. Where indicated, $100 \mu \mathrm{M}$ sodium pyrophosphate $(P P i)$ and $1 \mu \mathrm{M}$ nigericin $(N I G)$ were added. Curve a Cells grown in SDM-79 medium, curve $b$ cells grown in Warren's medium. Note that the higher activity was found in cells grown in SDM-79 medium. Scale bars A $1 \mu \mathrm{m}$; $300 \mathrm{~nm}$; C $150 \mathrm{~nm}$
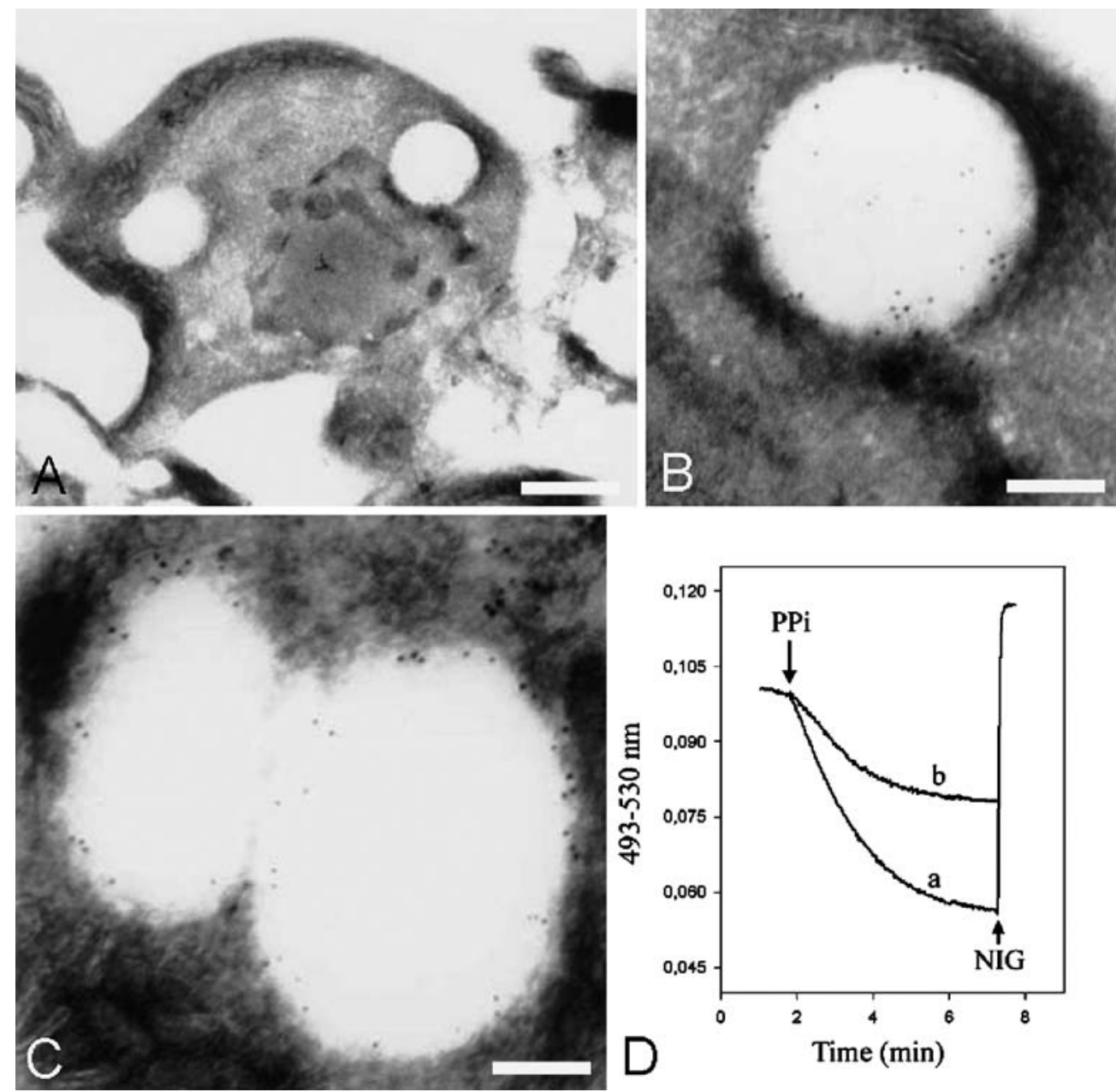

amazonensis (Ueda-Nakamura et al. 2002), are seen in different trypanosomatids during different phases of their cell cycles. These changes are possibly triggered by modifications in the surrounding environment in which the cells reside, i.e., phagolysosomes of macrophages and blood in the vertebrate host, and the digestive tract in the invertebrate host. These changes suggest that organellar plasticity in trypanosomatids is a natural condition for the establishment of infection.

Structural differences are relevant for the mechanisms that control acidity within acidocalcisomes. For instance, the mechanism required to maintain the internal $\mathrm{pH}$ of small structures such as vesicles of the endocytic pathway or acidocalcisomes in trypanosomatids has been unclear. Given the small and variable internal volumes of these vesicles, and, consequently, the small number of $\mathrm{H}^{+}$needed to achieve $\mathrm{pH}$ values between 5 and 6 , it is unlikely that such careful regulation is mediated by controlling the number of $\mathrm{H}^{+}$pumps per vesicle and it is possible that acidification may be regulated by alterations affecting the ion permeability characteristics of these organelles (Fuchs et al. 1989). This is particularly important for some parasites such as T. cruzi (Miranda et al. 2000), T. brucei (Rodrigues et al. 1999a), and L. donovani (Rodrigues et al. $1999 \mathrm{~b})$ that possess acidocalcisomes of very small di- mensions $(<200 \mathrm{~nm}$ diameter). Changes in the size of acidocalcisomes would affect the requirements for proton pumps and this could be the reason for the different proton pumping activity found in Leishmania grown under different culture conditions (Fig. 8D). The finding of spherical acidocalcisomes with an empty matrix $12 \mathrm{~h}$ after transferring cells from SDM-79 medium to Warren's medium suggest a decrease of the storage capability of the acidocalcisomes in cells grown in Warren's medium. These changes in the structural organization of the acidocalcisomes could be explained by the lack in the structural support in the matrix of the organelle. The lack of cations in the central portion of the matrix of the acidocalcisomes could lead to a collapse of the organelle, causing changes in the architecture of acidocalcisomes in cells grown in Warren's medium.

The finding of iron in acidocalcisomes of promastigotes grown in Warren's medium reinforces the hypothesis that these organelles are involved in iron homeostasis in trypanosomatids. The finding of a different elemental composition of the acidocalcisomes of the same parasite grown under different culture conditions indicates that the composition of the culture medium influences the composition of the acidocalcisomes. 
Fig. 9A-D Three-dimensional reconstruction shows the degree of ramification of acidocalcisomes from cells grown in different culture media. A Threedimensional reconstruction of serial sections of cells grown in SDM-79 medium showing spherical acidocalcisomes. B Three-dimensional reconstruction of serial sections of cells grown in Warren's medium showing branched acidocalcisomes. C, D Stereopairs of acidocalcisomes of cells grown in Warren's medium
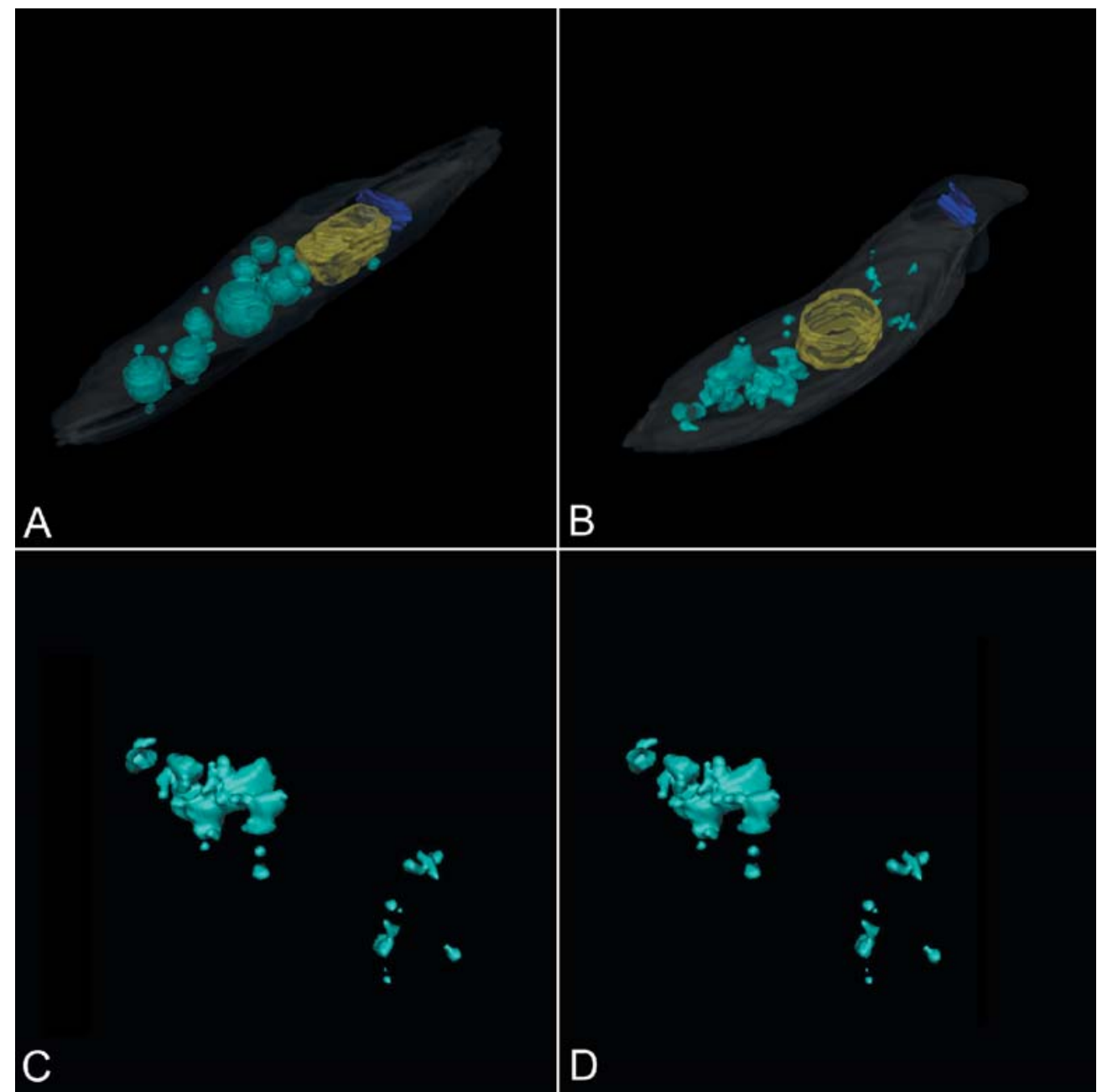

The reversal of the morphology and elemental composition of the acidocalcisomes in cells transferred to Warren's medium and back again to SDM-79 medium suggests that the acidocalcisome characteristics are associated to particular properties of the culture medium and are not the result of a shock caused by changing the culture medium. Measurements of the osmolality in both media showed a similar value of around 300 mosmol (data not shown).

In conclusion, our findings demonstrate that depending on the environmental conditions, L. amazonensis promastigotes can display acidocalcisomes with different structural, and chemical properties. This is, therefore, direct evidence for the role of the acidocalcisomes in environmental sensing and adaptation of the parasites to environmental changes.

Acknowledgements We thank Dr. Philip A. Rea for gifts of polyclonal antibodies and Dr. Mark H. Ellisman for the use of the facilities at the National Center for Microscopy and Image Research at San Diego, supported by United States Public Health Service Grant NIHRR0450. This work was supported in part by grants from Conselho Nacional de Desenvolvimento TecnológicoCNPq, Conselho de Aperfeiçoamento de Profissionais de Ensino Superior-CAPES, Fundação Carlos Chagas Filho de Amparo a
Pesquisa do Estado do Rio de Janeiro-FAPERJ, Fundação de Amparo a Pesquisa do Estado de São Paulo-FAPESP, Programa de Núcleos de Excelência-PRONEX, Deutsche Forschungsgemeinshaft (to H.P.), and the National Institutes of Health (AI-23259 to R.D.).

\section{References}

Attias M, Vommaro RC, De Souza W (1996) Computer aided three-dimensional reconstruction of the free-living protozoan Bodo sp. (Kinetoplastida: Bodonidae). Cell Struct Funct 21: 297-306

Brun R, Schönenberg M (1979) Cultivation and in cloning of procyclic culture forms of Trypanosoma brucei in a semi-defined medium. Acta Trop 36:289-292

Correa A, Andrade L, Soares M (2002) Elemental composition of acidocalcisomes of bloodstream forms of Trypanosoma cruzi. Parasitol Res 88:875-880

Cunha-e-Silva N, Atella GC, Porto-Carreiro I, Morgado-Dias J, Pereira MG, De Souza W (2002) Isolation and characterization of a reservosome fraction of Trypanosoma cruzi. FEMS Microbiol Lett 214:7-12

De Souza W (2002) Special organelles of some pathogenic protozoa. Parasitol Res 88:1013-1025

De Souza W, Porto-Carreiro I, Miranda K, Cunha-e-Silva N (2000) Two special organelles found in Trypanosoma cruzi. An Acad Bras Cienc 1:421-432 
Docampo R, Moreno SNJ (1999) Acidocalcisome: a novel $\mathrm{Ca}^{2+}$ storage compartment in trypanosomatids and apicomplexan parasites. Parasitol Today 15:443-448

Docampo R, Moreno SNJ (2001) The acidocalcisome. Mol Biochem Parasitol 33:151-159

Docampo R, Scott DA, Vercesi AE, Moreno SNJ (1995) Intracellular $\mathrm{Ca}^{2+}$ storage in acidocalcisomes of Trypanosoma cruzi. Biochem J 310:1005-1012

Drozdowicz YM, Shaw M, Nishi M, Striepen B, Liwinski HA, Roos DS, Rea PA (2003) Isolation and characterization of TgVP1, a type $\mathrm{I}$ vacuolar $\mathrm{H}^{+}$-translocating pyrophosphatase from Toxoplasma gondii. The dynamics of its subcellular localization and the cellular effects of a diphosphonate inhibitor. J Biol Chem 278:1075-1085

Fahrenbach WH (1984) Continuous serial sectioning for electron microscopy. J Electron Microsc Tech 1:387-393

Fuchs R, Schmid S, Mellman I (1989) A possible role for $\mathrm{Na}^{+}, \mathrm{K}^{+}-$ ATPase in regulating ATP-dependent endosome acidification. Proc Natl Acad Sci U S A 86:539-543

Guilbride DL, Englund TP (1998) The replication mechanisms of kinetoplast DNA networks in several trypanosomatid species. J Cell Sci 111:675-679

Handman E (2000) Cell biology of Leishmania. Adv Parasitol 44:1-27

Hessler D, Young SJ, Carragher BO, Martone M, Hinshaw JE, Milligan RA, Masliah E, Whitaker M, Lamont S, Ellisman MH (1992) Programs for visualization in three-dimensional microscopy. Neuroimage 1:55-67

LeFurgey A, Ingram P, Blum J (2001) Compartmental responses to acute osmotic stress in Leishmania major result in rapid loss of $\mathrm{Na}^{+}$and $\mathrm{Cl}^{-}$. Comp Biochem Physiol 128:385-394

Lemercier G, Espiau B, Ruiz F, Vieira M, Luo S, Balltz T, Docampo R, Bakalara N (2003) A pyrophosphatase regulating polyphosphate metabolism in acidocalcisomes is essential for Trypanosoma brucei virulence in mice. J Biol Chem 279:34203425

Lingelbach K (2001) Life in vacuoles: a strategy for parasite survival. Int. J Parasitol 31:5-6

Lu HG, Zhong L, Chang KP, Docampo R (1997) Intracellular $\mathrm{Ca}^{2+}$ pool content and signaling and expression of a calcium pump are linked to virulence in Leishmania mexicana amazonesis amastigotes. J Biol Chem 272:9464-9473

Marchesini N, Ruiz F, Vieira M, Docampo R (2002) Acidocalcisomes are functionally linked to the contractile vacuole of Dictyostelium discoideum. J Biol Chem 277:8146-8153

Miranda K, Benchimol M, Docampo R, De Souza W (2000) The fine structure of acidocalcisomes in Trypanosoma cruzi. Parasitol Res 86:373-384

Miranda K, Rodrigues C, Catisti R, Vercesi A, Plattner H, Hentschel J, De Souza W, Docampo R (2004) Acidocalcisomes of Phytomonas françai possess distinct morphological characteristics and contain iron. Microsc Microanal (in press)

Moreno B, Urbina J, Oldfield E, Bailey B, Rodrigues C, Docampo $\mathrm{R}(2000){ }^{31} \mathrm{P}$ NMR spectroscopy of Trypanosoma brucei, Trypanosoma cruzi, and Leishmania major. Evidence for the presence of high levels of condensed inorganic phosphates. J Biol Chem 275:28356-28362

Palmgren MG (1991) Acridine orange as a probe for measuring $\mathrm{pH}$ gradients across membranes: mechanisms and limitations. Anal Biochem 192:316-321

Parsons M, Ruben L (2000) Pathways involved in environmental sensing in trypanosomatids. Parasitol Today 16:56-62

Porto-Carreiro I, Attias M, Miranda K, De Souza W, Cunha e Silva NL (2000) Trypanosoma cruzi epimastigote endocytic pathway: cargo enters the cytostome and passes through an early endo- somal network before storage in reservosomes. Eur J Cell Biol 79:858-869

Rodrigues C, Scott D, Docampo R (1999a) Characterization of a vacuolar pyrophosphatase in Trypanosoma brucei and its localization to acidocalcisomes. Mol Cell Biol 19:7712-7723

Rodrigues C, Scott D, Docampo R (1999b) Presence of a vacuolar $\mathrm{H}^{+}$-pyrophosphatase in promastigotes of Leishmania donovani and its localization to a different compartment from the vacuolar $\mathrm{H}^{+}$-ATPase. Biochem J 340:759-766

Ruiz F, Marchesini N, Seufferheld M, Govingee, Docampo R (2001a) The polyphosphate bodies of Chlamydomonas reinhardtii possess a proton pumping pyrophosphatase and are similar to acidocalcisomes. J Biol Chem 276:46196-46203

Ruiz F, Rodrigues C, Docampo R (2001b) Rapid changes in polyphosphate content within acidocalcisomes in response to cell growth, differentiation and environmental stress in Trypanosoma cruzi. J Biol Chem 276:26114-26121

Sarafian V, Kim Y, Poole, RJ, Rea PA (1992) Molecular cloning and sequence of cDNA encoding the pyrophosphate-energized vacuolar membrane proton pump of Arabdopsis thaliana. Proc Natl Acad Sci U S A 89:1775-1779

Scott DA, Docampo R (1998) Two types of ATPases are involved in the acidification of acidic compartments in Trypanosoma cruzi. Biochem J 331:583-589

Scott D, Docampo R, Dvorak JA, Shi S, Leapman R (1997) In situ compositional analysis of acidocalcisomes in Trypanosoma cruzi. J Biol Chem 272:28020-28029

Scott DA, De Souza W, Benchimol M, Zhong L, Lu H, Moreno S, Docampo R (1998) Presence of a plant-like proton-pumping pyrophosphatase in acidocalcisomes of Trypanosoma cruzi. J Biol Chem 273:22151-22158

Seufferheld M, Vieira MCF, Ruiz FA, Rodrigues CO, Moreno SNJ, Docampo R (2003) Identification in bacteria of organelles similar to acidocalcisomes of unicellular eukaryotes. J Biol Chem 278:29971-29978

Ueda-Nakamura T, Sampaio MCR, Cunha-e-Silva NL, TraubCseko Y, De Souza W (2002) Expression and processing of megasome cysteine proteinases during Leishmania amazonensis differentiation. Parasitol Res 88:332-337

Urbina J, Moreno B, Vierkotter S, Oldfield E, Payares G, Sanoja C, Bailey B, Yan W, Scott D, Moreno S, Docampo R (1999) Trypanosoma cruzi contains major pyrophosphate stores, and its growth in vitro and in vivo is blocked by pyrophosphate analogs. J Biol Chem 274:33609-33615

Vercesi AE, Docampo R (1996) Sodium-proton exchange stimulates $\mathrm{Ca}^{2+}$ release from acidocalcisomes of Trypanosoma brucei. Biochem J 315:265-270

Vercesi AE, Moreno S, Docampo R (1994) $\mathrm{Ca}^{2+} / \mathrm{H}^{+}$exchange in acidic vacuoles of Trypanosoma brucei. Biochem J 304:227233

Vercesi AE, Grijalba M, Docampo R (1997) Inhibition of $\mathrm{Ca}^{2+}$ release from Trypanosoma brucei acidocalcisomes by 3,5 dibutyl-4-hydroxytoluene: role of the $\mathrm{Na}^{+} / \mathrm{H}^{+}$exchanger. Biochem J 328:479-482

Vercesi AE, Rodrigues CO, Catisti R, Docampo R (2000) Presence of a $\mathrm{Na}^{+} / \mathrm{H}^{+}$exchanger in acidocalcisomes of Leishmania donovani and their alkalinization by anti-leishmanial drugs. FEBS Lett 473:203-206

Warren LG (1960) Metabolism of Schizotrypanum cruzi. Chagas. I. Effect of culture age and substrate concentration on respiratory rate. J Parasitol 46:529-539

Young SJ, Royer SM, Groves PM, Kinnamon JC (1987) Threedimensional reconstruction from serial micrographs using an IBM PC. J Electron Microsc Tech 6:207-215 